RESEARCH LETTER -Professional Development

\title{
A European questionnaire-based study on population awareness and risk perception of antimicrobial resistance
}

\section{Elena-Alexandra Alexa (Oniciuc) ${ }^{1,2}$, Eleni Likotrafiti ${ }^{1,3}$, Alberto Garre ${ }^{4}$, Lorena Ruiz ${ }^{5}$, Miguel Prieto ${ }^{1,6}$ and Avelino Alvarez-Ordóñez ${ }^{1,6, *}$}

${ }^{1}$ Department of Food Hygiene and Technology, Universidad de León, León, Spain, ${ }^{2}$ Faculty of Food Science and Engineering, Dunarea de Jos University of Galati, Galati, Romania, ${ }^{3}$ Department of Food Science and Technology, Laboratory of Food Microbiology, International Hellenic University, Thessaloniki, Greece, ${ }^{4}$ Food Microbiology, Wageningen University \& Research, Wageningen, the Netherlands, ${ }^{5}$ Department of Microbiology and Biochemistry of Dairy Products, Instituto de Productos Lácteos de Asturias - Consejo Superior de Investigaciones Científicas, Villaviciosa, Spain and ${ }^{6}$ Institute of Food Science and Technology, Universidad de León, León, Spain

*Corresponding author: Tel: +34 987291274; E-mail: aalvo@unileon.es

One sentence summary: A European study on the public awareness of antimicrobial use, including the sources and routes for AMR spread and transmission to consumers via food and other reservoirs.

Editor: Beatrix Fahnert

\begin{abstract}
To tackle antimicrobial resistance (AMR) is of outmost importance for the general population to understand the severity and the relevance of different routes of transmission. Respondents of different age groups, educational and occupational backgrounds, area of living, diet and household composition participated in an online survey with questions concerning socio-demographics, personal use of antibiotics, awareness, general knowledge, sources of information, behavior and attitude toward antibiotics, and risk perception on antibiotics and AMR. Descriptive and logistic regression analyses were carried out. A total of 1252 respondents, mainly from EU, participated in the survey. About $57.7 \%$ declared they consumed antibiotics in the last year and some misguided behaviors were identified, especially for those not having a food- or health-related background, who more frequently failed in giving the right answer to uncontroversial true/false questions (ANOVA, $P<0.05)$. The youngest respondents were less confident on the information received from traditional media $(\mathrm{OR}=0.425)$, the national government $(\mathrm{OR}=0.462)$, and consumer organizations $(\mathrm{OR}=0.497)$, while they frequently obtained information from social networks and online media, which could therefore be exploited as a channel for educational campaigns targeting this population group. New measures, strategies and policy agenda at a European level aimed at improving awareness on AMR among targeted community groups must be taken into consideration.
\end{abstract}

Keywords: antimicrobial resistance; awareness; knowledge; consumption; antibiotics; risk perception 


\section{INTRODUCTION}

The advent of antibiotics in the 20th century revolutionized the clinical management of microbial infections, both in human and animal medicine, followed by a sharp decrease in mortality rates (Aminov 2010). However, soon after their introduction, antimicrobial resistance (AMR) inevitably emerged, threatening their efficacy (Aminov 2010; Wright 2010; Laxminarayan et al. 2013). Nowadays, AMR represents a great concern, which requires urgent attention, as we may be soon facing an era where antibiotics will be ineffective to combat several types of bacteria causing life-threatening infections, with a consequent increase in healthcare-related costs (Davies and Davies 2010; Ventola 2015; Malik and Bhattacharyya 2019).

The general population should actively participate in control programs undertaking actions aimed at limiting AMR spread and maintaining antibiotics' effectiveness (Lee et al. 2013; WHO 2015). Indeed, different educational campaigns on AMR targeting the general population, or healthcare and veterinary professionals, have been already conducted in some countries through different channels of communication (Afzal Khan, Banu and Reshma 2013; Mazińska, Strużycka and Hryniewicz 2017). Although such campaigns will likely not suffice alone to tackle the complex issue posed by AMR, they have already played an important role in the battle against it (Collignon et al. 2018). For instance, implementation of strategic action plans led to a reduction in antibiotic usage and AMR rates in Sweden over a 10 years period, for which analyzing trends in antibiotic consumption and AMR was crucial to identify knowledge gaps and prioritize certain interventions (Mölstad et al. 2008).

Surveys requested by the European Commission monitor the public use of and knowledge about antibiotics at European Union (EU) level (European Commission 2013, 2016, 2018). Notably, antibiotics usage dropped down from $40 \%$ to $32 \%$ between 2009 and 2018 (European Commission 2016, 2018). Awareness on AMR amongst the general population largely depended on the country of origin and on the availability of specialized information via different means. Also, Europeans prioritized actions at global and EU level (27\%) over those at national or regional level (19\%) or at individual or family level (19\%) (European Commission 2018). In addition, several studies based on surveys have targeted issues related to antibiotics and AMR among medical students (Afzal Khan, Banu and Reshma 2013; Scaioli et al. 2015; Jana et al. 2016), physicians (Labi et al. 2018) or the general population of certain cities (Mazińska, Strużycka and Hryniewicz 2017; Prigitano et al. 2018; Yusef et al. 2018). For instance, the Polish population showed a more cautious usage of antibiotics at household level after the implementation of the European Antibiotic Awareness Day, although consumption and inappropriate use of antibiotics was still highly prevalent (Mazińska, Strużycka and Hryniewicz 2017).

In this study, the results of a questionnaire-based survey gathering information regarding the general knowledge on antibiotics and AMR and the perceived risks to human health linked to antibiotic resistant bacteria (ARB) are presented. Specifically, it addresses the public awareness on antimicrobial use, including the sources and routes for AMR spread and transmission to consumers via food and other reservoirs. Information on the channels through which consumers' perception is influenced, and on changes of attitudes and values-driven decisions regarding antibiotics use and food consumption is also analyzed.

\section{METHODS}

Study design

A cross-sectional study based on an online questionnaire was carried out between April and May 2018. Participation was anonymous, independent and without any compensation, as each respondent self-completed the questionnaire using the Google forms platform. The purpose of the questionnaire was explained beforehand to the respondents, who were asked to complete the questionnaire by selecting the options that felt most appropriate. The questionnaire was distributed across multiple channels including: (i) focal points of the European Food Safety Authority; (ii) University communities of the coauthor's institutions and (iii) networks of academic collaborators, family and friends.

\section{Characteristics of the questionnaire}

The questionnaire was designed based on a literature review of the field and following discussions between the co-authors. The first part of the questionnaire was designed to identify socio-demographic factors which might influence the answers or opinions of respondents. The second part, divided into six subsections, addressed questions related to: (i) personal use of antibiotics, (ii) awareness, (iii) general knowledge, (iv) sources of information, (v) behavior and attitude towards antibiotics and (vi) risk perception about antibiotics and AMR. A summary of the questions included in the questionnaire is provided in Table S1 (Supporting information).

\section{Statistical analysis}

Statistical analysis was carried out using $\mathrm{R}$ version 3.4 .3 and IBM SPSS Statistics for Windows Version 24.0. Several descriptive analyses were carried out (e.g. box-plots, histograms, pairs plots) to visualize the distribution of the variables and to identify possible relationships between them. A logistic regression analysis was applied to identify the most significant $(P$-values $<0.05)$ socio-demographic factors related to antibiotics usage, awareness, general knowledge, sources of information, behavior and attitude towards antibiotics, and risk perception. Results were expressed as Odds Ratio (OR) with 95\% Confidence Intervals (CI). Plots were generated using the R packages ggplot2 and ggmap (Kahle and Wickham 2013). The data set and computer codes used are available upon request.

\section{RESULTS}

\section{Socio-demographics}

A total of 1252 respondents mainly from the EU countries (86.4\%) completed the survey, with majority from Spain (23.6\%), Greece $(11.7 \%)$, Iceland (8.2\%), Finland (7.7\%) and Croatia (6.8\%) (Fig. S1, Supporting Information). Additional socio-demographic information is presented in Table 1.

\section{Personal use of antibiotics}

The majority of respondents took antibiotics in the last year (57.7\%), 87.6\% of them following a medical prescription. Among those who took antibiotics, $96.6 \%$ believe that they must adhere to the medical instructions on dosage and treatment periods, while $2.8 \%$ would stop the treatment once they feel better. Most respondents do not take antibiotics after the expiration date 
Table 1. Socio-demographic features of the respondents

\begin{tabular}{|c|c|c|}
\hline Demographic feature & Respondents & $\begin{array}{l}\text { Percentage } \\
(\%)\end{array}$ \\
\hline \multicolumn{3}{|l|}{ Gender } \\
\hline Male & 363 & 29 \\
\hline Female & 889 & 71 \\
\hline \multicolumn{3}{|l|}{ Age group } \\
\hline $18-34$ & 653 & 52 \\
\hline $35-54$ & 461 & 37 \\
\hline $55-74$ & 138 & 11 \\
\hline \multicolumn{3}{|l|}{ Continent } \\
\hline EU & 1082 & 86 \\
\hline Non- EU & 170 & 14 \\
\hline \multicolumn{3}{|l|}{ Area of living } \\
\hline $\begin{array}{l}\text { City (with more than } 100000 \\
\text { inhabitants) }\end{array}$ & 705 & 56 \\
\hline Town (with 10 000-100 000 inhabitants) & 325 & 26 \\
\hline $\begin{array}{l}\text { Small town/village/rural (town or village } \\
\text { with less than } 10000 \text { inhabitants or } \\
\text { outside of significant population } \\
\text { centers) } \\
\text { Education }\end{array}$ & 222 & 18 \\
\hline Doctorate & 267 & 21 \\
\hline Master's/Professional degree & 441 & 35 \\
\hline Bachelor's degree & 291 & 23 \\
\hline $\begin{array}{l}\text { High school/secondary school graduate } \\
\text { with diploma/qualifications }\end{array}$ & 204 & 16 \\
\hline Technical/Vocational training/diploma & 42 & 3 \\
\hline Obligatory education & 6 & 0,5 \\
\hline No schooling completed & 1 & 0,1 \\
\hline \multicolumn{3}{|l|}{ Food- or health-related education } \\
\hline Yes & 876 & 70 \\
\hline No & 376 & 30 \\
\hline \multicolumn{3}{|l|}{ Food- or health-related profession } \\
\hline Yes & 814 & 65 \\
\hline No & 438 & 35 \\
\hline \multicolumn{3}{|l|}{ Diet } \\
\hline No special diet & 1089 & 87 \\
\hline $\begin{array}{l}\text { Special diet (for example pescetarian, } \\
\text { vegetarian, plant-based diet or other) } \\
\text { Household composition }\end{array}$ & 163 & 13 \\
\hline $\begin{array}{l}\text { Married/domestic partnership and at } \\
\text { least one child }\end{array}$ & 402 & 32 \\
\hline $\begin{array}{l}\text { Married/domestic partnership-adults } \\
\text { only }\end{array}$ & 312 & 25 \\
\hline Single adult and at least one child & 35 & 3 \\
\hline Single adult only & 503 & 40 \\
\hline
\end{tabular}

(89.4\%), while some do not check that information or are not concerned about it.

Notably, those respondents which completed tertiary education will less likely take antibiotics (OR $=0.119,95 \%$ CI: 0.028 0.510), and those not having a food- or health-related education background will more likely consume them (OR $=2.823,95 \%$ CI: 1.212-6.576). Regarding household composition, married couples, regardless of whether they have children (OR $=2.289,95 \%$ CI: $1.266-4.141)$ or not (OR $=2.783,95 \%$ CI: 1.516-5.109), more frequently take antibiotics with medical prescription than single adults (Table S2, Supporting Information).
Table 2. Responses to different statements included in the Q7 from the survey for addressing the actual knowledge of respondents $(n=1252)$

\begin{tabular}{lc} 
Statement & $\begin{array}{c}\text { Correct response, } \mathrm{n} \\
(\%)\end{array}$ \\
\hline S1. Antibiotics kill viruses & False, $1155(92.3 \%)$ \\
S2. Antibiotics kill bacteria & True, $1179(94.2 \%)$ \\
S3. Antibiotics should be used to treat flu & False, 1166 (93.1\%) \\
S4. Antibiotics often result in side effects & True, $905(72.3 \%)$
\end{tabular}

such as diarrhea

S5. Antibiotics should not be taken without True, 1200 (95.8\%) consulting a doctor

S6. Antibiotics counteract symptoms of $\quad$ False, 967 (77.2\%) fever

S7. Antibiotics are needed to protect

True, 640 (51.1\%)

animal health

S8. Overuse of antibiotics in animals may

lead to the antibiotics becoming ineffective

to treat infections in animals or humans

S9. Antibiotic use in animal husbandry is regulated by legislation

S10. Antibiotics are not used in animal husbandry, even if animals are sick

S11. Antibiotics are used to cure infections in farm animals

S12. Antibiotics are used to prevent

infections in farm animals

S13. Antibiotics are used in the EU to

stimulate the growth of farm animals

S14. Antibiotics cannot be used in non-EU

countries to stimulate the growth of farm

animals

S15. Antibiotics used on farm animals are different from those used on people

True, 1153 (92.1\%)

True, 859 (68.6\%)

False, $1215(97 \%)$

True, $1056(84.3 \%)$

True, $737(58.9 \%)$

False, 957 (76.4\%)

False, 1084 (86.6\%)

False, 992 (79.2\%)

\section{General knowledge about antibiotics}

The general knowledge of the population on different AMR-related issues was evaluated through 15 true/false statements (Table 2). Most respondents were aware that antibiotics are not effective for killing viruses (92.3\%), that should not be used to treat flu (93.1\%) and that should not be taken without a medical prescription (95.8\%). A slightly lower number were aware that antibiotics do not counteract fever symptoms and often result in side effects such as diarrhea (77.2 and $72.3 \%$, respectively). Less awareness was shown on the use of antibiotics at farm settings (Table 2). Having a food- or health-related educational or professional background was associated with greater general knowledge (Analysis of variance (ANOVA), $P<0.05$ ), with mean knowledge scores in the true/false questions of $12.5 \pm 1.68$ and $12.5 \pm 1.73$ positive answers, respectively, as compared to their counterparts (mean scores of $11.6 \pm 1.89$ and $11.7 \pm 1.81$, respectively). Respondents from South-Eastern Europe (score: $11.9 \pm 2.02$ ) showed less general knowledge than those from Northern (score: $12.6 \pm 1.49)$ and Western Europe (score: $12.5 \pm 1.52$ ), respectively (Fig. 1). 

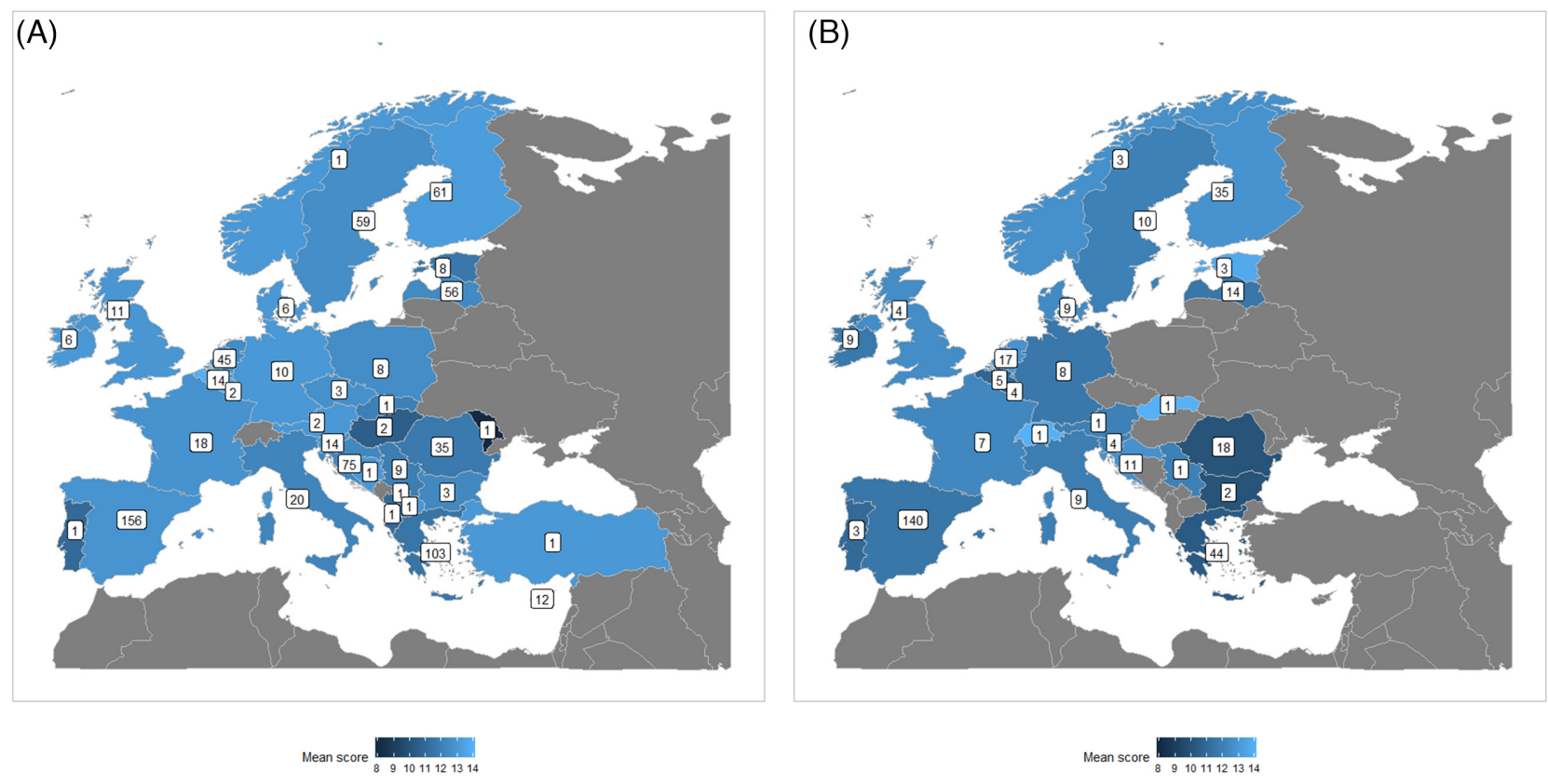

Figure 1. Geographical differences in general knowledge on antibiotics and AMR for respondents (A) with a food- or health-related job and (B) without a food- or health-related job. Color scale directly reflects the general knowledge score obtained, while numbers reflect the total number of respondents per country.

\section{Awareness about antibiotics and ARB}

The vast majority of respondents had previously heard about ARB (97.8\%) and considered that inappropriate use of antibiotics at household level (97.9\%), farms (97.8\%) and clinical settings (95.7\%) were likely to cause AMR. Respondents also linked consumption of food containing antibiotics (88.9\%), inappropriate activities at pharmaceutical industries (85.6\%) and other non-specified causes (82.3\%) to AMR. Besides, $62.1 \%$ of respondents considered the natural emergence in the human body as a likely cause for AMR.

The oldest age group felt better informed about AMR than the $18-34$ (OR $=0.164,95 \% \mathrm{CI}: 0.064-0.422)$ and $35-54$ age groups (OR $=0.465,95 \%$ CI: 0.176-1.226). Contrarily, those living in big cities (>100000 habitants) felt less informed about antibiotics $(\mathrm{OR}=0.111,95 \% \mathrm{CI}: 0.025-0.489)$ than those living in small towns; those not having a food- or health-related educational background felt less informed about antibiotics (OR $=0.284,95 \% \mathrm{CI}$ : 0.149-0.540) and AMR (OR =0.190, 95\% CI: 0.109-0.331); and those not working in a food- or health-related field felt less informed about AMR (OR $=0.254,95 \%$ CI: 0.144-0.447), also obtaining a lower general knowledge score (Fig. 2).

Notably, respondents from the 18 to 34 age group $(\mathrm{OR}=1.878$, 95\% CI: $1.249-2.823$ ) or with tertiary education (OR $=1.765,95 \%$ CI: 1.277-2.438) considered natural emergence of AMR in the human body more likely to occur.

\section{Sources of information}

The majority of respondents declared having received information about ARB in humans in the last year (78.3\%), coming from TV or radio news (60.6\%), food- and human healthrelated websites $(49.8 \%)$, health professionals $(43.8 \%)$, social media (28.9\%) and family (15.8\%), among other less frequent sources. About $52.9 \%$ had also received information about ARB in farm animals, mainly from TV or radio news (48.1\%), foodand human health-related websites (45.9\%), health professionals $(39.9 \%)$ and social media (26\%). However, only $43.2 \%$ had received information about $A R B$ in food, commonly from foodand human health-related websites (43.4\%), TV or radio news (39.7\%), health professionals (37.5\%) and social media (20\%). Overall, the sources used depended on age and professional background. Notably, the oldest age group more frequently used traditional media (newspapers, radio and television), while younger respondents gave more importance to social networks (Fig. 3A).

Respondents expressed a high confidence on the information received from scientists (96.6\%), European institutions (97.2\%), national and European agencies (92\%) and other food- and health-related workers/institutions (72.1\%). Family and friends (67.5\%), consumer organizations (66.7\%) and farmers $(66 \%)$ inspired slightly less confidence, while environmental protection groups (60.7\%), food manufacturers $(60.6 \%)$ and traditional mass media (television, radio and newspaper)s (52.9\%) were identified as the less trustworthy sources (Fig. 3B).

The youngest group (18-34 years) was less confident on the information received from traditional mass media (OR $=0.425$, 95\% CI: 0.279-0.647), the national government ( $O R=0.462,95 \%$ CI: $0.264-0.808)$ and consumer organizations (OR $=0.497,95 \%$ CI: 0.307-0.803) (Fig. 3B). Respondents living in large cities were more confident in the European institutions (OR $=2.125,95 \%$ CI: 1.352-3.340) while those who finished tertiary education felt more confident about most sources of information, including mass media (OR $=1.831,95 \%$ CI: $1.301-2.577$ ) and others (Table S2, Supporting Information). The educational and professional background also had some influence on the confidence shown on different information sources (Fig. 3B).

\section{Behavior and attitude}

The belief that AMR can affect their own health (92.2\%) encouraged some respondents to change behavior or attitudes toward antibiotics and/or food in the last year (51.4\%). These behavioral changes include talking to family or friends (32.2\%), seeking more information $(29 \%)$, changing eating habits $(18.8 \%)$ or even 


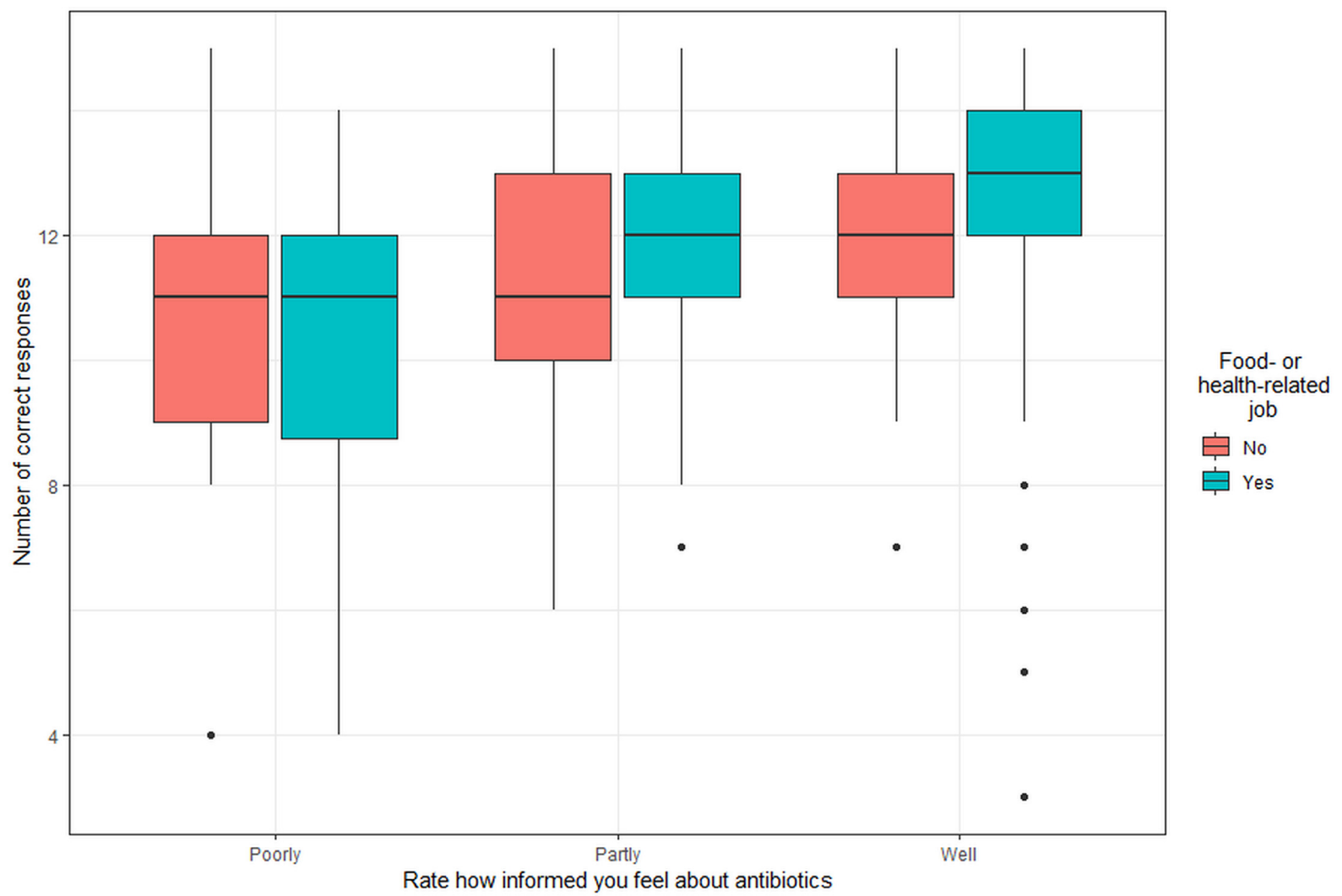

Figure 2. General knowledge on antibiotics and AMR and perceived awareness about antibiotics as a function of whether respondents had a food- or health-related professional background or not.

talking with food and health-related authorities about AMR in farming (7\%).

Some respondents $(29.5 \%)$ take into consideration whether animals are produced or not with the use of antibiotics when buying meat products. However, almost half $(43.3 \%)$ of them did not know where to check such information and $24.4 \%$ did not care about this. Interestingly, almost $78.5 \%$ would be willing to pay more money for foodstuffs produced without the use of antibiotics. Besides, women (OR $=1.838,95 \%$ CI: $1.370-2.465)$ showed an increased willingness to pay more money for meat products obtained from animals produced without antibiotics, as compared to male respondents.

The oldest age group is more likely to change dietary habits in response to the information received on antibiotics and AMR than those from the 18 to 34 (OR $=0.429,95 \%$ CI: $0.282-0.654$ ) and 35 to 55 age group (OR $=0.525,95 \%$ CI: 0.345-0.798). Similarly, those following a special diet (mainly vegetarians) more frequently changed their eating habits in the last year than those following regular diets (OR $=0.410,95 \%$ CI: $0.285-0.588$ ). Regarding the priority level given to different measures aimed at tackling AMR, the majority declared that legislation on antibiotics use should be more restrictive (89.6\%), and that consumers should be better informed on AMR-related risks (86.9\%). Respondents would be also willing to help farmers to shift to organic farming (86.2\%) and to provide more funding for research related to the replacement of ineffective antibiotics (85.3\%). Lower importance was given to providing financial incentives to agrifood industries $(77.4 \%)$ or to the organization of pressure campaigns by consumer organizations (68.1\%). These opinions were significantly influenced by several socio-demographic factors. Respondents not working in food- or health-related institutions gave less priority to providing financial incentives to industries (OR $=0.554,95 \%$ CI: $0.376-0.816)$ or farmers (OR $=0.499,95 \%$ CI: 0.308-0.809), while women were more inclined to help farmers shift production modes (OR $=1.798,95 \% \mathrm{CI}: 1.231-2.625)$ than males. Older respondents or followers of special diets were more interested in participating in consumer campaigns than their respective counterparts (Table S2, Supporting Information). Finally, respondents with lower education levels consider more important to better inform consumers on the risks posed by ARB than those with a tertiary education (OR $=0.340,95 \%$ CI: $0.166-$ 0.698).

\section{Risk perception}

A relatively high proportion of respondents felt strongly threatened by AMR (48\%), while $46.6 \%$ felt barely threatened and $5.3 \%$ did not feel threatened at all. The vast majority believed that giving antibiotics to healthy animals in order to prevent illness (89.5\%) or to stimulate growth (87.6\%) could contribute to AMR. Besides, they considered that treating unhealthy or weak animals, with or without a veterinary prescription $32.1 \%$ and $84.7 \%$, respectively), might also contribute to AMR spread.

Overall, the food chain AMR transmission route was considered as a relevant one, including transmission from farm animals to farmers $(63.7 \%)$, consumers $(60.1 \%)$, meat handlers (59.7\%) or veterinarians (48.8\%). AMR transmission to humans through direct contact with live farm animals (44.7\%) or pets (32.5\%) was also considered, as well as the transmission of AMR in clinical settings $(49.8 \%)$ or through person-to-person contact (37.8\%). 
(A)

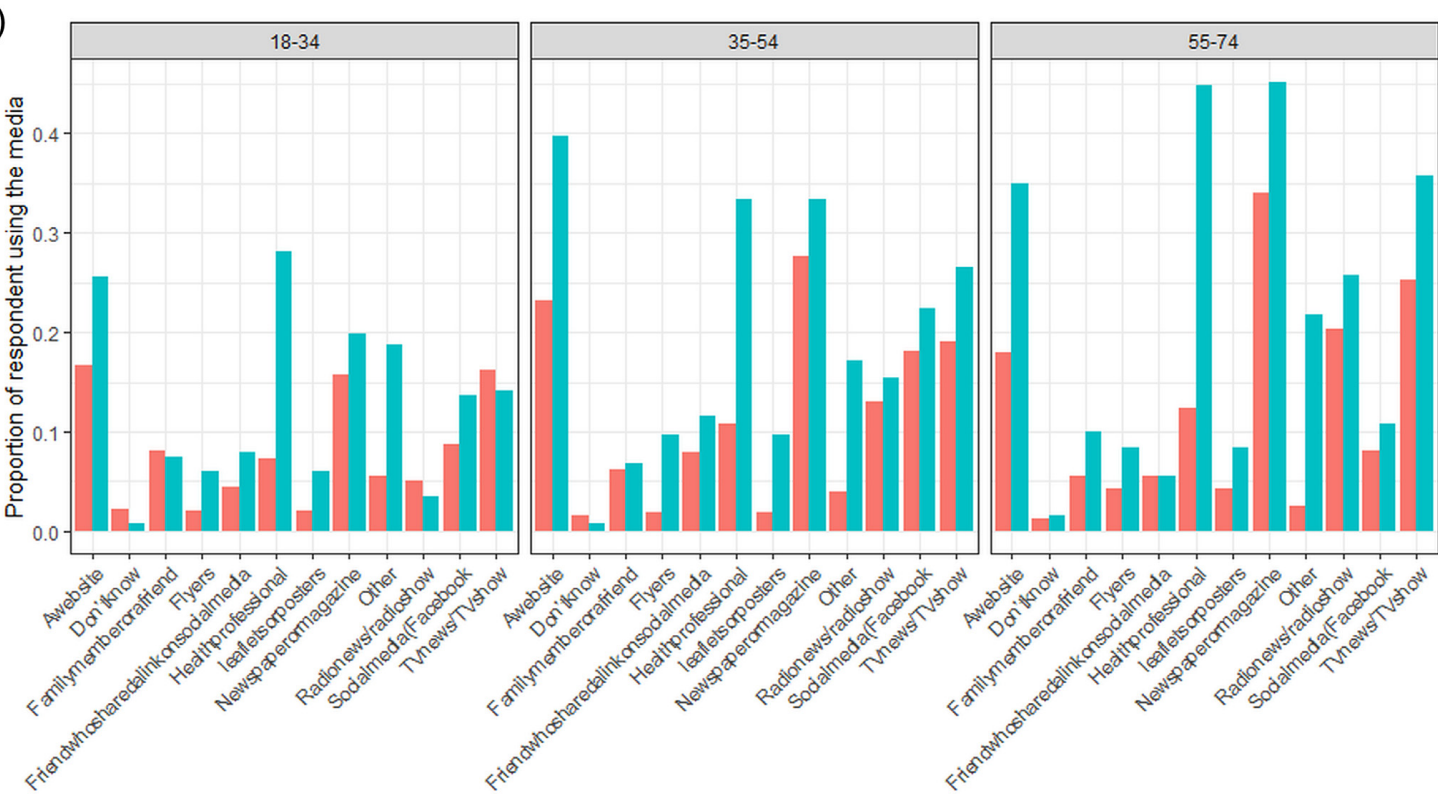

Food- or health-related

job No

(B)

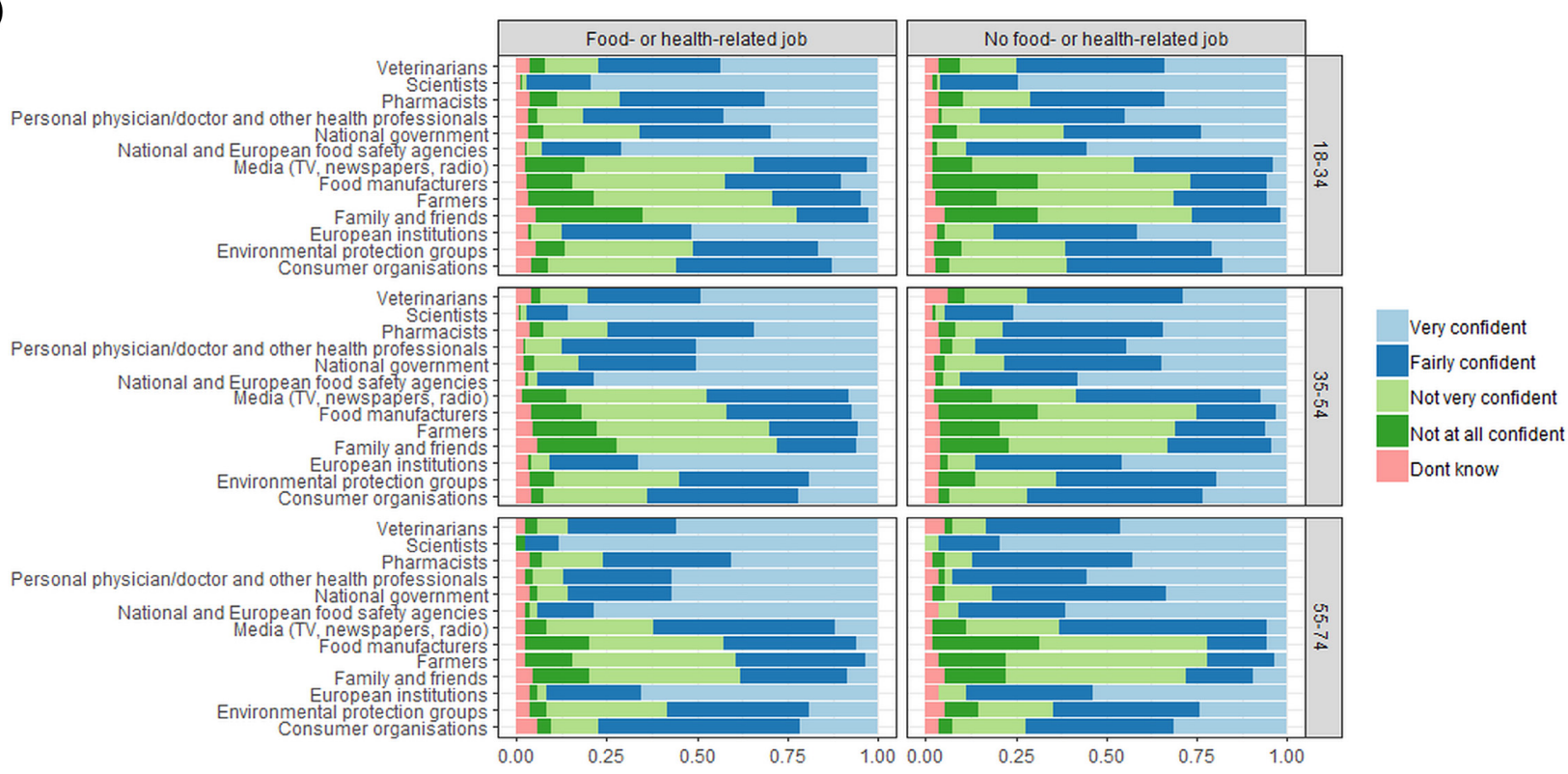

Figure 3. Variations in the sources of information and population trust on them. (A), Sources of information used according to age and professional background. (B), Confidence of the respondents on different sources of information according to age and professional background.

Overall, respondents not having a food- or health-related job considered a less relevant risk factor treating weak or unhealthy animals with antibiotics without a veterinary prescription (OR $=0.484,95 \%$ CI: 0.306-0.766); and older respondents considered a less relevant risk factor using antibiotics as growth promoters than respondents from 3the 5 to 54 age group (OR $=2.475,95 \%$ CI: $1.271-4.819)$. On the other hand, they were more worried about treating unhealthy or weak animals with prescribed antibiotics than respondents from 18the to 34 age group (OR $=0.564,95 \%$ CI: $0.372-0.856$ ). Women considered more relevant the transmission from farm animals to veterinarians (OR $=1.596,95 \% \mathrm{CI}: 1.228-2.075)$, and to meat (OR $=1.544,95 \% \mathrm{CI}: 1.180-2.022)$; and gave a higher importance to AMR transmission in clinical settings
$(\mathrm{OR}=1.460,95 \% \mathrm{CI}: 1.121-1.902)$ or through the manipulation of rare meat $(\mathrm{OR}=1.461,95 \% \mathrm{CI}$ : 1.111-1.922) (Table S2, Supporting Information). Finally, the majority (76\%) believed than the benefits of antibiotics outweigh by far their associated risks. This view was influenced by the general knowledge of the population on antibiotics and AMR, with those respondents showing a better general knowledge on the topic giving a higher weight to the benefits of antibiotics $(P<0.05)$ (Fig. 4). 


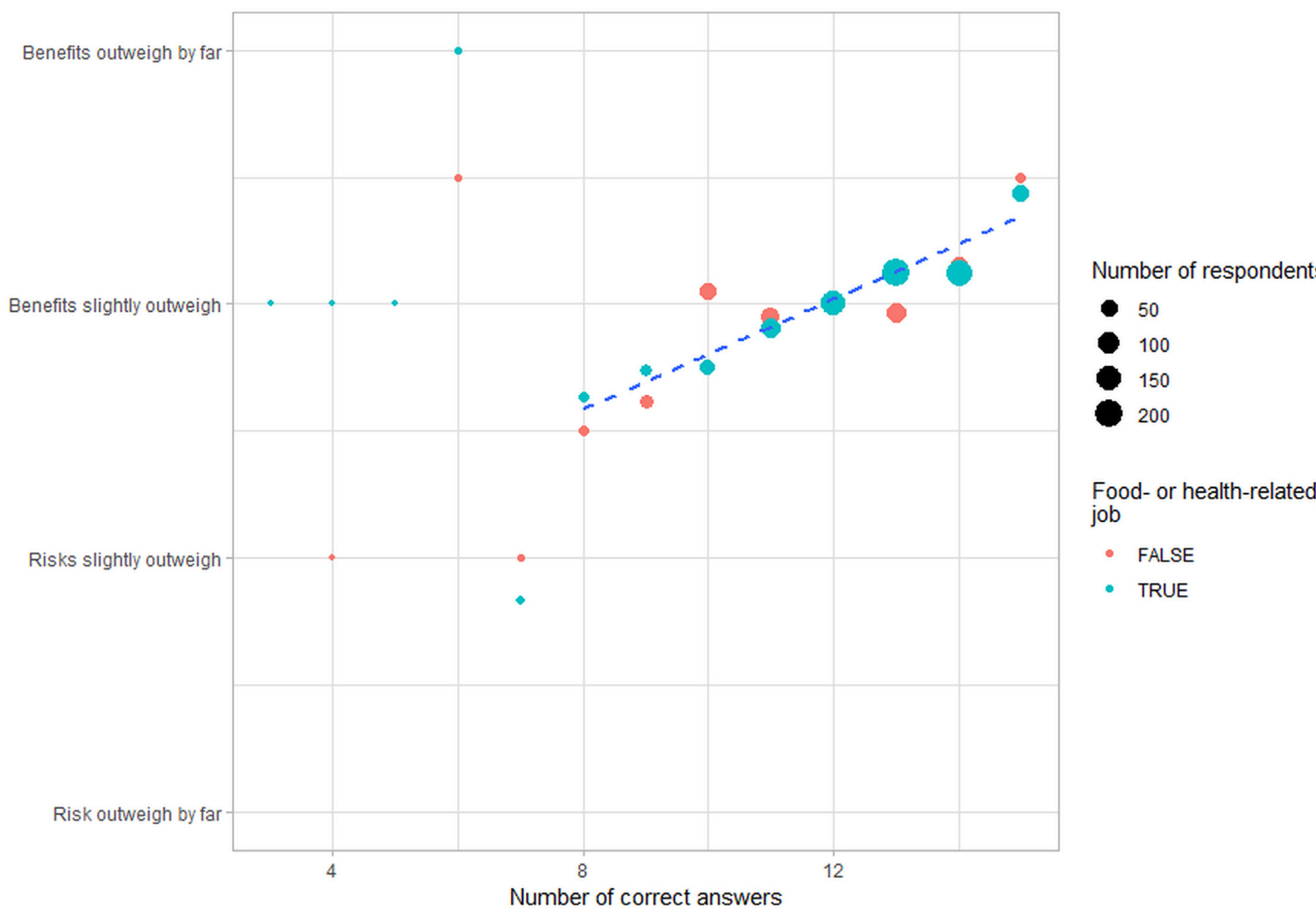

Figure 4. Relationship between the population general knowledge of AMR and the estimated risk-benefit ratio according to professional background. The size of the data points indicates the number of respondents.

\section{DISCUSSION}

This study aimed at assessing the awareness, attitude and behavior of the general population on issues related to antibiotics use and the emergence and transmission of AMR, paying also attention to the role that the food chain may play. To the best of our knowledge, this is the first European study investigating such a broad range of topics (i.e. antibiotics consumption and mode of use, awareness and general knowledge, sources of information and consumers' confidence on them, behavior and attitude towards antibiotics and risk perception) amongst the general population using an online questionnaire to increase its scope. Indeed, although there were a number of constraints in the composition of the sampled population (i.e. higher participation in some countries, such as Spain and Greece; overrepresentation of respondents with high-educational level and with food- or health-related educational and professional background), this represents a very comprehensive survey involving more than 1200 participants, mainly from the EU. The fact that a significant proportion of the people who completed the survey were professionally orientated represents a potential bias. However, this, on the other hand, offered the opportunity of comparing the perception, opinions and behaviors of two population groups with similar number of respondents: (i) respondents without higher education or professional background related to food and health and (ii) respondents with higher education or professional background related to food and health. The results obtained allowed us identify some misperceptions and knowledge gaps among the general population.
Educational campaigns and surveys addressing effective ways to combat AMR have been previously conducted. In a previous initiative focused on EU Member States almost 30000 respondents from different socio-demographic groups were face-to-face interviewed in their mother tongue on behalf of the Directorate-General for Health and Food Safety (European Commission 2016). The volume of data obtained is currently being used to analyze if the action plan against AMR is successfully tackling such issue but also to spot areas where it would be helpful to develop new strategies or which require improvements (European Commission 2016). Our study provides additional information to the EU surveys.

Almost two-thirds (57.7\%) of the respondents took antibiotics during the last year, which was higher compared to a previous study carried out in Poland (38\%) (Mazińska, Strużycka and Hryniewicz 2017) or to consumption data reported in the 2013 (35\%) (European Commission 2013), 2016 (34\%) (European Commission 2016) and 2018 (32\%) Eurobarometers (European Commission 2018). A slightly higher consumption of antibiotics has been observed among students of a Medicine school in Italy (45.6\%) (Scaioli et al. 2015) or respondents of 12 World Health Organization (WHO) Member States (65\% in the past 6 months) (WHO 2015). However, most recent studies often targeted specific groups, such as physicians (Labi et al. 2018) or medical and nursing students (Afzal Khan, Banu and Reshma 2013; Scaioli et al. 2015; Jana et al. 2016), while very few studies have assessed antibiotics use in the general community. Interestingly, the likelihood of consuming more antibiotics is higher among those not having received tertiary education or training in a food- or health-related field, probably associated with knowledge gaps 
leading to an irresponsible behavior. This fact evidences that further investment in education can contribute to diminish antibiotic consumption among the general population.

Despite the relatively high rates of antibiotic consumption observed, most consumers are quite responsible regarding antibiotics use, not taking them without consulting the doctor and strictly following the general practitioner guidelines. Only a small proportion (8\%) declared to procure antibiotics without prescription, which is comparable to findings in the 2018 Eurobarometer (European Commission 2018). It is important to highlight that inadequate knowledge of the consumer or the prescriber may contribute to overprescribing, further contributing to AMR (Huttner et al. 2010).

Overall, the general knowledge of respondents was relatively high, with a mean general knowledge score of $12.2 \pm 1.79$ positive answers out of 15 . Incorrect answers were mostly related to the use of antibiotics for animal health maintenance and animal husbandry. Higher awareness was shown about the fact that antibiotics are ineffective against viruses (92.3\%) than that previously reported among European respondents (43\%) (European Commission 2018) and respondents from WHO Member States (34\%) (WHO 2015). This misconception regarding antibiotics effectivity to treat viral infections has been highlighted in several studies among the general public (McNulty et al. 2007; European Commission 2016, 2018; Gaarslev et al. 2016; Davis et al. 2017). The level of education and the educational background were key factors influencing the ability of respondents to answer correctly the 15 true/false statements, this being demonstrated also in Puchner and Zacharisen (2002) and Hsiao et al. (2006). Our results also evidence that educational campaigns are needed in certain EU countries as differences were observed in the level of knowledge between respondents from Northern, Central and Southern Europe.

In general, the youngest respondents and those without a food- or health-related education or employment were the collectives with the lowest confidence in their knowledge on antibiotics and AMR, further evidencing the need to develop educational contents targeting these particular population sectors.

The current study gathered data on AMR-related hot topics and on the sources through which the general population was exposed to them. Respondents were frequently exposed to information related to AMR in humans but were less aware about AMR in farm animals and, especially, in foods. In terms of information channels, significant differences were observed among age groups as oldest respondents trusted more on traditional media (newspapers, printouts, radio and television), while younger respondents more frequently acquired information from online media, reflecting a generational change in information channels. It could be that older people have less access to online media or that they are less confident on such sources. Indeed, previous studies have revealed that the use of online media as a source of information increased 1.27 times the likelihood of becoming misinformed about AMR (Jacob et al. 2018). A higher confidence was expressed on the information coming from scientists, national and European agencies, and different related workers/institutions. Less confidence was expressed on the information obtained from national governments, family and friends and others (Table S2, Supporting Information). These findings agree with studies conducted among the Polish and Italian population indicating that doctors, pharmacists and nurses are the most reliable sources of information on antibiotics (Napolitano et al. 2013; Mazińska, Strużycka and Hryniewicz 2017) and with results of the Eurobarometer survey (European Commission 2018). In our study, in general, the youngest and those with a lower education level showed less confidence in most channels of information, and, as such, measures aimed at reinforcing the trust in the most damaged information channels should target these population groups.

Overall, respondents felt majorly threatened by AMR as compared to other previous reports. In our study, 92.2\% considered that AMR may have an impact on their health, while only half of the medical students (54\%) addressed in a previous survey considered this to be the case (Prigitano et al. 2018). Similarly, in a study conducted at a tertiary care institution in the Caribbean, $82 \%$ of the respondents believed that AMR is an important global issue but only $53 \%$ believed that they might be directly affected (Nicholson et al. 2018). Interestingly, hospital-based doctors considered that AMR was an important issue in their daily practice, over outpatient-based doctors in ambulatory care or physicians who practice in both areas (65\% vs. 39\% and 35\%, respectively) (Nicholson et al. 2018).

When rating potential causes for the emergence of AMR, the highest score was given to using antibiotics to prevent illness in healthy animals or as growth promotors. In addition, several AMR transmission routes involving farm animals were identified as relevant. Moreover, a significant number of respondents declared they had changed their eating habits, seeked information on AMR in farming or even talked to food safety agencies, as a result of their concern about the topic. Even, around one third of the respondents take into consideration when buying a meat product in the market whether the animal has been produced without the use of antibiotics, and the majority of them, especially women, are willing to pay more money for those products obtained from animals raised without antibiotics, highlighting the need for action programs focused at reducing antibiotics use in animal farming.

The AMR issue is an urgent matter and actions must be taken to confront it in a One Health approach (Flasche and Atkins 2018; Van Puyvelde, Deborggraeve and Jacobs 2018; Walsh 2018). The prioritization of actions among the respondents was in accordance to the respondents' background or particular interests (e.g. those working in a food-related field would be more willing to provide financial support to farmers and food industry stakeholders).

The respondents considered that the benefits of antibiotics outweighed by far the risks, with those ones showing a better general knowledge on the topic giving a higher weight to the benefits. Interestingly, the rest of the population over-estimated the risks over the benefits, probably because they only had access to news referring to negative aspects of the use of antibiotics. This fact evidences that it is also important to include information on the benefits of antibiotics, when responsibly used, in educational campaigns focused at improving the public awareness on antibiotics and AMR.

\section{CONCLUSIONS}

The irrational use of antibiotics is being recognized as the main factor involved in the emergence and spread of resistant bacteria. The present study highlights some gaps in the level of knowledge about antibiotics and ARB amongst the general European population and evidences that there are still some misguided attitudes and behaviors, especially for those respondents not having a food- or health-related background. Further strategies must therefore include measures aimed at improving awareness among targeted community groups on the appropriate use of antibiotics, with additional educational campaigns 
that could be beneficial for reducing antibiotics misuse at household level. Social networks and online media exploitation as a channel to transmit information to the youngest population represents an opportunity but has to be considered with caution as it can come with a price on how to correctly discern valuable information from fake news and other sources of misinformation.

\section{SUPPLEMENTARY DATA}

Supplementary data are available at FEMSLE online.

\section{AUTHORS' CONTRIBUTIONS}

EAA, EL, MP and AAO designed the survey. EAA, EL and LR wrote the initial draft of the manuscript and AG, EAA and AAO were involved in data analysis. Several versions circulated between the authors until they agreed on the final version of the manuscript. All authors read and approved the manuscript.

\section{ACKNOWLEDGMENTS}

EAA and EL are research fellows funded by the EU-FORA mobility program of the European Food Safety Authority. AG is grateful to the Fundación Seneca for awarding him a postdoctoral grant (20900/PD/18). LR is a postdoctoral fellow supported through a Juan de la Cierva Postdoctoral Trainee Program of the Spanish Ministry of Economy and Competitiveness (grant, IJCI-201523196). The authors would like to thank the respondents for their participation.

\section{FUNDING}

This work was supported by Ministerio de Economia y Competitividad, under Grant number AGL2016-78 085-P, which provided the necessary funds to accomplish the research. The funder did not contribute to the design of the study, the collection, analysis and interpretation of the data and writing of the manuscript.

Conflict of interest. None declared.

\section{REFERENCES}

Afzal Khan AK, Banu G, Reshma KK. Antibiotic resistance and usage-A survey on the knowledge, attitude, perceptions and practices among the medical students of a southern Indian teaching hospital. J Clin Diagnostic Res 2013;7:1613-6.

Aminov RI. A brief history of the antibiotic era: lessons learned and challenges for the future. Front Microbiol 2010;1:1-7.

Collignon P, Beggs JJ, Walsh TR et al. Anthropological and socioeconomic factors contributing to global antimicrobial resistance: a univariate and multivariable analysis. Lancet Planet Heal 2018;2:e398-405.

Davies J, Davies D. Origins and Evolution of Antibiotic Resistance. Microbiol Mol Biol Rev 2010;74:417-33.

Davis M, Liu T-L, Taylor Y et al. Exploring Patient Awareness and Perceptions of the Appropriate Use of Antibiotics: A MixedMethods Study. Antibiotics 2017;6:1-12.

European Commission. Special Eurobarometer 407 Report Antimicrobial Resistance. 2013.

European Commission. Special Eurobarometer 445 Report Antimicrobial Resistance. 2016.

European Commission. Special Eurobarometer 478 Report Antimicrobial Resistance. 2018.
Flasche S, Atkins KE. Balancing Benefits and Risks of Antibiotic Use. J Infect Dis 2018;218:1351-3.

Gaarslev C, Yee M, Chan G et al. A mixed methods study to understand patient expectations for antibiotics for an upper respiratory tract infection. Antimicrob Resist Infect Control 2016;5:19.

Hsiao F-Y, Lee J-A, Huang W-F et al. Survey of medication knowledge and behaviors among college students in Taiwan. Am J Pharm Educ 2006;70:30.

Huttner B, Goossens H, Verheij T et al. Characteristics and outcomes of public campaigns aimed at improving the use of antibiotics in outpatients in high-income countries. Lancet Infect Dis 2010;10:17-31.

Jacob G, James EK, Brittany A et al. Media use and antimicrobial resistance misinformation and misuse: survey evidence of information channels and fatalism in augmenting a global health threat. Cogent Med 2018;5:1-15.

Jana A, Jana A, Majumdar A et al. Practical knowledge and perception about antibiotic usage and resistance: a questionnaire-based study. Int J Sci Study 2016;4:89-95.

Kahle D, Wickham H. ggmap: spatial visualization with ggplot2. RJ 2013;5:144-61.

Labi A-K, Obeng-Nkrumah N, Bjerrum S et al. Physicians' knowledge, attitudes, and perceptions concerning antibiotic resistance: a survey in a Ghanaian tertiary care hospital. BMC Health Serv Res 2018;18:1-12.

Laxminarayan R, Duse A, Wattal C et al. Antibiotic resistance-the need for global solutions. Lancet Infect Dis 2013;13:1057-98.

Lee C-R, Cho IH, Jeong BC et al. Strategies to minimize antibiotic resistance. Int J Environ Res Public Health 2013;10:4274-305.

Malik B, Bhattacharyya S. Antibiotic drug-resistance as a complex system driven by socio-economic growth and antibiotic misuse. Sci Rep 2019;9:9788.

Mazińska B, Strużycka I, Hryniewicz W. Surveys of public knowledge and attitudes with regard to antibiotics in Poland: did the European Antibiotic Awareness Day campaigns change attitudes? Gupta V (ed). PLoS One 2017;12:e0172146.

McNulty CAM, Boyle P, Nichols T et al. The public's attitudes to and compliance with antibiotics. J Antimicrob Chemother 2007;60:163-8.

Mölstad S, Erntell M, Hanberger $\mathrm{H}$ et al. Sustained reduction of antibiotic use and low bacterial resistance: 10-year followup of the Swedish Strama programme. Lancet Infect Dis 2008;8:125-32.

Napolitano F, Izzo MT, Di Giuseppe G et al. Public knowledge, attitudes, and experience regarding the use of antibiotics in Italy. PLoS One 2013;8:e84177.

Nicholson A, Tennant I, White L et al. The knowledge, attitudes and practices of doctors regarding antibiotic resistance at a tertiary care institution in the Caribbean. Antimicrob Resist Infect Control 2018;7:1-9.

Prigitano A, Romanò L, Auxilia F et al. Antibiotic resistance: Italian awareness survey 2016. J Infect Public Health 2018;11:30-4.

Puchner TC, Zacharisen MC. A survey of antibiotic prescribing and knowledge of penicillin allergy. Ann Allergy, Asthma Immunol 2002;88:24-9.

Van Puyvelde S, Deborggraeve S, Jacobs J. Why the antibiotic resistance crisis requires a One Health approach. Lancet Infect Dis 2018;18:132-4.

Scaioli G, Gualano MR, Gili R et al. Antibiotic Use: A crosssectional survey assessing the knowledge, attitudes and practices amongst students of a school of medicine in Italy. PLoS One 2015;10:1-12. 
Ventola CL. The antibiotic resistance crisis. Pharm Ther 2015;40:277-83.

Walsh TR. A one-health approach to antimicrobial resistance. Nat Microbiol 2018;3:854-5.

WHO. Antibiotic Resistance: Multi-Country Public Awareness Survey. 2015.
Wright GD. Q \& A: antibiotic resistance: where does it come from and what can we do about it ? BMC Biol 2010;8:1-6.

Yusef D, Babaa AI, Bashaireh AZ et al. Knowledge, practices \& attitude toward antibiotics use and bacterial resistance in Jordan: a cross-sectional study. Infect Dis Heal 2018;23:33-40. 